\title{
Introduction à l'hydrovolcanologie
}

\begin{abstract}
Michel DETAY
Schematics Ltd, 7/F., Bonham Centre, 79-85 Bonham Strand, Sheung Wan, Hong Kong, SAR China - e-mail : mdetay@yahoo.com

RÉSUMÉ. - L'hydrovolcanologie profonde s'intéresse au rôle joué par la molécule d'eau (sous forme hydroxylée ou moléculaire) dans l'individualisation des magmas par fusion partielle au sein du manteau et/ou de la croûte terrestre, leur remontée dans la plomberie magmatique, jusqu'à l'exsolution-fragmentation grande responsable des dynamismes éruptifs explosifs. L'hydrovolcanologie superficielle couvre le champ des phénomènes paravolcaniques pré et postéruptifs : solfatares, fumerolles, sources chaudes, flux géothermiques, qui représentent un indicateur important en terme de prévisions volcanologiques. Elle permet de mieux comprendre la dynamique des lacs de cratère et des lacs de rift, souvent sursaturés en $\mathrm{CO}_{2}$ d'origine volcanique, susceptibles d'éruptions limniques. L'hydrovolcanologie présente une perspective nouvelle au sein des sciences hydrotechniques où l'eau est à la fois l'élément déclencheur, voire le catalyseur, d'éruptions explosives majeures, mais aussi un indicateur précieux en matière de prévision et de gestion du risque.
\end{abstract}

Mots-clés : hydrovolcanologie, hydrogéologie des grandes profondeurs, volcanologie, hydrologie

\section{Hydrovolcanology: an introduction}

\begin{abstract}
Our knowledge of the physics of how volcanoes work has expended enormously over the past 20 years, as have our methods of studying volcanic processes. In seeking to understand volcanic behavior, volcanologists call on a diversity of physics subdisciplines, including fluid dynamics, thermodynamics, solid mechanics, hydrovolcanology, ballistics and acoustics, to name a few. Deep hydrovolcanology describes the water involvement in magma generation and segregation through partial melting into the Earth's crust and/or mantle, magma upward migration in the volcanic plumbing system and exsolution-fragmentation in the subsurface. As the magma rises towards the surface the confining pressure decreases, the volatiles gradually exsolve from the magma forming the gas bubbles which are distributed throughout the liquid. It is the connecting together of a network of these bubbles that ultimately causes the continuous body of liquid to break apart or fragment into a spray of droplets or clots suspended in the gas. In magmas, typically $95-99 \%$ of the 'mass' of material erupted is liquid rock - at most the gas accounts for only a few percent of the weight; but that small amount of gas represents a very large 'volume' as it expands to atmospheric pressure, and is fundamentally important in producing explosive eruptions. Continued rise of the magma leads to further exsolution of gas and growth of gas bubbles through diffusion, decompression and bubble coalescence. The relative importance of each process depends on the amount of volatiles (gas - mostly water) present in the magma, the magma composition and the magma rise speed. Surface hydrovolcanology is involved in pre and post-eruptive paravolcanic activity such as solfataras, fumaroles, hydrothermal heat and water fluxes. It also focuses on limnic eruptions, otherwise referred to as a lake overturns, a type of natural disaster in which dissolved carbon dioxide $\left(\mathrm{CO}_{2}\right)$ suddenly erupts from deep volcanic lakes, suffocating wildlife, livestock and humans. Hydrovolcanology presents a new perspective within hydrotechnical sciences where water is a trigger in magma generation and segregation, magma rising and eruption style. Water appears to be a most valuable indicator for volcanic hazard assessment and mitigation, short-term eruption prediction, and volcanic risk management. Understanding the physical behavior of volcanoes is critical for assessing the hazards posed to the ever-increasing populations living in close proximity to active volcanoes, and thus for mitigating the risk posed by those hazards.
\end{abstract}

Mots-clés : hydrovolcanology, deep hydrogeology, volcanology, hydrology

\section{INTRODUCTION}

Les sciences hydrotechniques s'intéressent à l'eau sous toutes ses formes et dans toutes les applications de la mécanique des fluides et de l'environnement. Nous présentons une dimension singulière de l'eau, peu connue et qui semble contre-intuitive, loin des applications hydrotechniques classiques, mais essentielle dans la dynamique du système Terre : l'hydrovolcanologie. Il s'agit d'une discipline nouvelle qui s'intègre dans la mécanique des fluides géologiques, à la frontière entre la volcanologie et l'hydraulique souterraine des grandes profondeurs.

Ce court article décrit brièvement l'importance de l'eau dans le volcanisme à diverses échelles, dans les profondeurs du manteau terrestre, lors de l'individualisation des magmas, puis lors de leur remontée vers la surface pour finir dans le phénomène éruptif explosif. Nous présentons aussi l'importance de l'eau dans les phénomènes paravolcaniques pré et postéruptifs. Notre objectif est de montrer que l'eau est un élément structurant en volcanologie. L'hydrovolcanologie offre une perspective nouvelle au sein des sciences hydrotechniques, permettant de décrire la physique du processus éruptif et de rendre compte de ses divers mécanismes par le rôle de l'eau et les lois physiques de l'hydraulique. L'eau sous diverses formes, dans ses manifestations hydrovolcaniques superficielles, est aussi un indicateur précieux en matière de prévision d'éruptions volcaniques et de gestion du risque. 


\section{I.1. Volcanologie}

La volcanologie est une science récente. La nature volcanique du Massif central n'a été reconnue que dans seconde moitié du XVIII ${ }^{\mathrm{e}}$ siècle. En 1902, l'éruption de la montagne Pelée (Martinique), faisant 29000 morts en quelques minutes, a représenté l'élément déclencheur de la nécessité de comprendre le phénomène volcanique. En 1950, H. Tazieff a fait découvrir les volcans au public grâce à ses films. En 1976, la célèbre polémique Allègre-Tazieff à propos de la Soufrière (Guadeloupe) fournit une caricature de la volcanologie française, du niveau d'ignorance des phénomènes volcaniques et de l'impréparation de nos institutions devant les crises volcaniques. Les éruptions volcaniques sont difficiles à étudier et leur comportement reste mal compris, malgré plusieurs dizaines d'années de recherches [Jaupart C., Kaminski E., (2006)]. Parfois dangereuses et destructrices, elles ne se prêtent pas toujours à l'observation directe et ont la fâcheuse habitude de détruire l'instrumentation mise en place sur les édifices volcaniques. Elles sont la cause de grandes catastrophes humaines et de changements climatiques importants.

La volcanologie est restée longtemps une science d'observation, essentiellement descriptive. Son vocabulaire et ses concepts sont souvent assez éloignés de l'explication physico-chimique des phénomènes impliqués. De très nombreux styles éruptifs ont ainsi été décrits : hawaïen, strombolien, vulcanien, péléen, plinien, fissural, islandais... pour finalement ne conserver que les quatre premiers. D'autres simplifications ont été proposées : l'opposition entre volcans rouges et gris, ou l'opposition entre les deux grands styles éruptifs (effusif et explosif). Cette dernière est la plus pertinente. En effet, récemment, la physique du processus éruptif et l'étude des volcans actifs ont mis en évidence qu'un édifice volcanique peut, au cours de sa vie, d'une éruption à l'autre, voire au cours d'une seule et même éruption, passer d'un style éruptif à un autre, d'une activité rouge à grise, d'une activité effusive à explosive. L'Eyjafjöll (Islande), par exemple, a bloqué l'espace aérien européen en 2010 en passant d'un style effusif à un style explosif [Detay M. (2010)]. De même la Soufrière de Montserrat, dans les Petites Antilles, a connu depuis 1995 pratiquement tous les styles éruptifs possibles. Les styles éruptifs ne sont donc pas caractéristiques d'un volcan, mais uniquement d'un moment donné de l'éruption. Le concept «volcan rouge », qui produit une lave fluide, par opposition au «volcan gris ", qui émet des produits pyroclastiques lors d'éruptions violentes, est également réducteur et trop simplificateur. Chaque style éruptif est associé à une typologie de produits éruptifs, mais un volcan ne développe pour ainsi dire jamais les mêmes types de lave au cours de sa période d'activité. Enfin, les scientifiques se sont longtemps focalisés sur le volume de gaz émis lors d'une éruption, mais ce qui est déterminant en volcanologie c'est la manière dont le gaz va s'individualiser lors de l'ascension du magma. La mise en perspective de l'importance des éléments volatils, et de l'eau en particulier (hydrovolcanologie), a permis une avancée significative dans la compréhension des mécanismes impliqués en volcanologie contemporaine. Elle permet notamment de préciser les quatre grands styles éruptifs.

\section{I.2. Hydraulique souterraine des grandes profondeurs}

L'étude des fluides géologiques, et l'analyse et la mise en équation de la physique du processus ont récemment permis de comprendre que les grands responsables des dynamismes éruptifs étaient les éléments volatils (les gaz), et en particulier l'eau. En effet, l'eau est présente en grande quantité dans les couches internes de la Terre. Il y a, au moins, l'équivalent d'une masse de l'ensemble de l'hydrosphère terrestre (masse océanique) présente dans le manteau (couche intermédiaire entre le noyau planétaire et la croûte terrestre, le manteau représente un peu plus de $80 \%$ du volume de la Terre et environ $65 \%$ de sa masse). L'eau est souvent impliquée dans les processus de fusion partielle à l'origine des magmas. En remontant des couches profondes, les éléments volatils dissous dans les magmas nouvellement formés vont s'exsolver, et provoquer la fragmentation du magma en approchant de la surface. L'exsolution individualise en profondeur des bulles de gaz microscopiques qui peuvent occuper, après décompression pendant la remontée du magma, jusqu'à $99 \%$ du volume éruptif en surface. Le paradoxe veut que ce soient de tout petits objets qui déterminent le devenir d'une colonne éruptive se développant à très grande échelle, sur des dizaines de kilomètres d'altitude (nuage pliniens et courants de densité pyroclastique), et susceptible d'avoir un impact global sur l'ensemble de la planète.

Ainsi, pour appréhender la dynamique des éruptions volcaniques, il faut s'intéresser à l'hydrovolcanologie, c'est-à-dire aux relations intimes entre la molécule d'eau et les processus physico-chimiques qui vont être à l'origine des magmas et conditionner leur ascension et les styles éruptifs. In fine, c'est la composition chimique des magmas, la quantité d'eau qui $\mathrm{y}$ est dissoute et la manière dont la phase gazeuse va s'individualiser qui contrôlent les régimes éruptifs. L'eau et les fluides géologiques jouent donc un rôle majeur dès le stade de la genèse du magma et tout au long de sa remontée. Selon le contexte géodynamique, le rôle de l'eau dans la genèse du magma est différent : majeur dans les cas de subductions, plus limité dans ceux de dorsales et de points chauds. Les magmas terrestres peuvent contenir jusqu'à $10 \%$ de leur poids en eau $\left(\mathrm{H}_{2} \mathrm{O}\right)$, et les gaz libérés par les volcans sont composés jusqu'à $90 \%$ d'eau. C'est le passage de l'eau à l'état gazeux lors de la remontée du magma qui est le grand responsable des divers dynamismes éruptifs sur notre planète.

\section{I.3. Planète océane ou planète volcan ?}

Depuis que les astronautes ont observé la Terre de l'espace, celle-ci a pris le nom poétique de «planète bleue » ou " planète océane », tant la présence d'eau y est dominante en surface : à $71 \%$, la Terre est recouverte d'océans. Mais ce que l'on a omis de considérer, c'est que les planchers océaniques sont majoritairement de nature volcanique. Alors que l'on dénombre environ 1500 volcans (dont environ 714 toujours actifs) depuis le début de l'Holocène (ces douze mille dernières années) sur les terres émergées, on estime à au moins 1,5 million le nombre d'édifices volcaniques présents sur le plancher océanique, soit mille fois plus. Par ailleurs, les océans renferment $53700 \mathrm{~km}$ de dorsale et $30000 \mathrm{~km}$ de zones de subduction, soit un dispositif volcanique majeur d'une longueur supérieure à deux fois le tour de la Terre [Detay M., Detay A.-M. (2013)]. Les circulations hydrothermales libérées au fond des océans participent à la constitution des ressources minérales du futur (nodules polymétalliques, encroûtements). Ils représenteraient un potentiel au moins égal aux ressources minérales terrestres. Vue sous cet angle, notre planète océane n'est pas bien loin d'être une planète volcan, d'autant que les roches d'origine magmatique sont largement prédominantes sur Terre.

L'eau est également impliquée dans la tectonique des plaques en imposant un saut brutal de viscosité de $10^{4}$ 
entre la lithosphère et le manteau. Notons que le manteau perd de l'eau par le volcanisme (env. $2.10^{12} \mathrm{~kg} / \mathrm{an}$ ), mais ce volume remis dans l'atmosphère et l'océan est compensé par le même volume d'eau introduit dans le manteau au niveau des $30000 \mathrm{~km}$ de zones de subduction. Le manteau terrestre hydraté permet l'existence d'une tectonique des plaques. La Terre dispose ainsi d'un régime stationnaire en matière de présence d'eau dans le manteau. D'autres planètes comme Vénus et Io, où il n'y a pas d'eau liquide susceptible de réhydrater le manteau, ont perdu définitivement leur eau par le volcanisme, et la tectonique des plaques s'y est arrêtée.

\section{HYDROVOLCANOLOGIE PROFONDE}

La présence de quantités importantes d'eau dans les grandes profondeurs des enveloppes terrestres est contre-intuitive. L'eau libre, qu'elle soit superficielle ou souterraine, est abondante à la surface de la Terre, mais elle est aussi présente en grandes quantités et sous diverses formes à très grande profondeur, jusqu'à la base du manteau, voire jusque dans le noyau [Bolfan-Casanova N. (2005) ; Dewaele A., Sanloup C. (2005)]. Dans les dix à vingt premiers kilomètres de la croûte terrestre, l'eau circule en empruntant un réseau de fissures et de fractures interconnectées. Même si la concentration des fluides aqueux est faible dans les profondeurs du manteau, le volume de celui-ci est tel que les quantités d'eau stockées sont très importantes, au moins équivalentes au volume d'eau visible en surface (hydrosphère) [Jolivet L., (2011)].

\section{II.1. Au moins la masse de l'hydrosphère dans le manteau terrestre}

L'eau est omniprésente à la surface, mais aussi dans la Terre profonde. Elle peut faire partie intégrante de la formule chimique de certains minéraux où l'ion $\mathrm{HO}^{-}$est intégré dans la structure cristalline telles les amphiboles, la lawsonite, la chlorite...) ; ces deux derniers minéraux contenant $14 \%$ d'eau. On peut également trouver de l'eau dans des minéraux dont la formule chimique n'en contient pas (minéraux nominalement anhydres - $M N A$ ), comme l'olivine et le quartz, sous forme de défauts ponctuels. Cette eau conditionne de manière drastique les propriétés physico-chimiques du manteau supérieur terrestre.

Les études géochimiques, géophysiques et pétrographiques mettent en évidence qu'il y a au moins l'équivalent de la masse d'eau de l'ensemble de l'hydrosphère $\left(1,4.10^{21} \mathrm{~kg}\right.$ d'eau), sous forme hydroxyle $\left(\mathrm{HO}^{-}\right)$ou moléculaire $\left(\mathrm{H}_{2} \mathrm{O}\right)$, dissous dans le manteau. Le cycle interne de l'eau déduit de ces études (cf. Figure 1) propose un bilan des quantités d'eau qui entrent dans le manteau (avec les plaques subduites) et qui en sortent (volcanisme d'arc et de dorsale). La teneur totale en eau de la Terre est estimée par les géochimistes à partir des scénarios de formation de la planète (entre $2,2 \cdot 10^{21} \mathrm{~kg}$ et $8,8 \cdot 10^{21} \mathrm{~kg}$ ). Connaissant la masse des océans actuels $\left(1,4.10^{21} \mathrm{~kg}\right)$, il apparaît que la Terre profonde est susceptible de renfermer entre 1,6 et 6,3 masses océaniques terrestres. S'agissant d'un sujet de recherche très actif qui dépasse notre propos, retenons qu'il y a au moins la masse d'un océan terrestre dans la Terre profonde ; les dernières estimations [Schmandt B et al., (2014)] seraient de trois masses océaniques terrestres.

\section{II.2. L'eau à l'origine des magmas primaires par fusion partielle}

Lorsque l'on s'intéresse aux couches internes de la planète, « le centre de la Terre ", l'image retenue par le grand public est généralement fausse. Contrairement à une idée reçue encore largement répandue, nous ne marchons pas sur un océan de magma. Il n'y a pas de « feu central». Les roches constitutives du manteau sont à l'état solide. La Terre silicatée n'est pas en fusion, il n'y a pas de magma liquide dans la Terre « normale ».

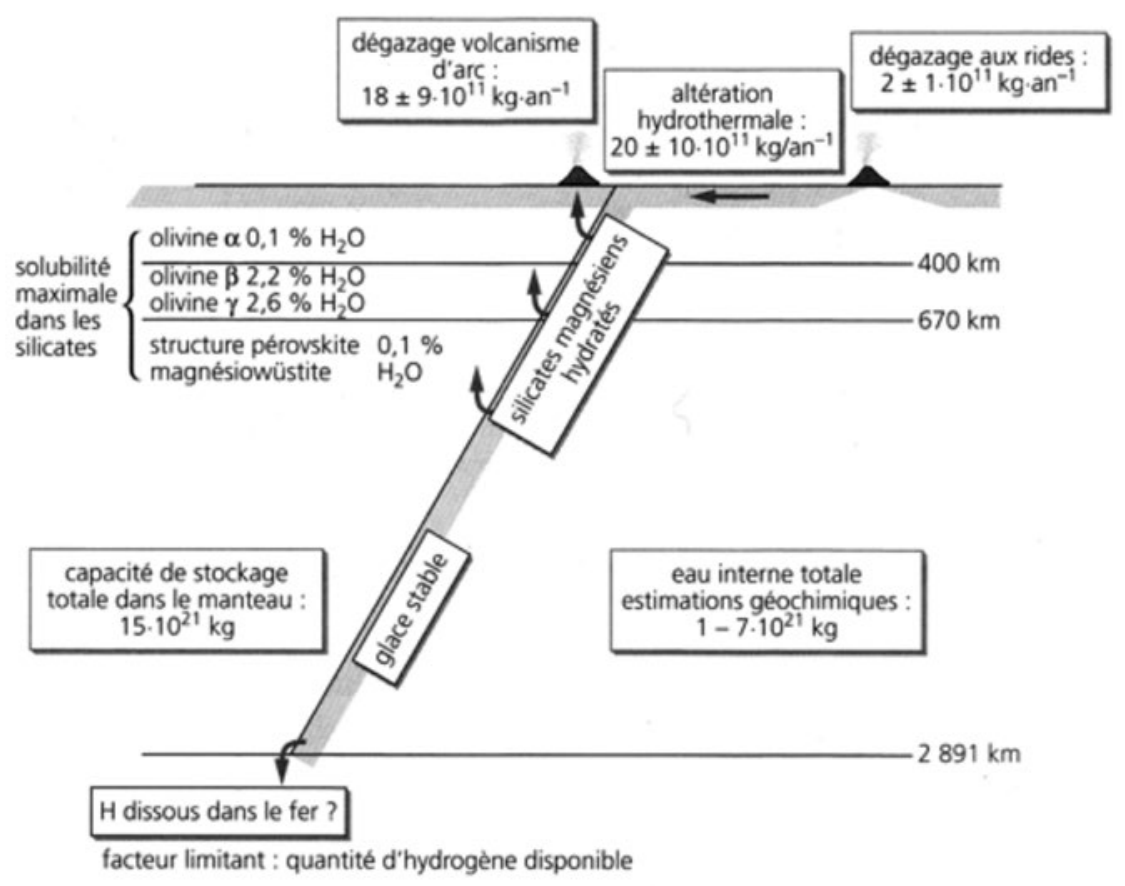

Figure 1 : Cycle interne de l'eau, bilan des flux d'eau entrant et sortant de la Terre profonde, d'après Dewaele A. et Sanloup C. (2005). 
Les travaux expérimentaux relatifs aux mécanismes de production de magmas mettent clairement en évidence la question essentielle de la composition du manteau. Le magma primaire s'individualise par fusion partielle à partir des roches constitutives du manteau, les péridotites [silicate de fer $(10 \%)$ et de magnésium $(90 \%)]$. Les lherzolites à spinelle et les lherzolites à grenat sont des péridotites supposées constituer le matériel mantellique primitif parce que leur composition chimique leur permet de produire un liquide basaltique lors d'une fusion partielle expérimentale. Par ailleurs, les propriétés sismiques et les masses volumiques de ces lherzolites sont compatibles avec les observations faites sur le manteau [Robert C., Bousquet R. (2013)]. Il apparaît clairement, sur un diagramme pression-température, que, dans les conditions normales, le géotherme ne croise pas le solidus du manteau lherzolitique (cf. Figure 2), il n'y a donc pas de fusion possible du manteau en condition "normale ». Bien que le phénomène soit encore mal compris, on pense que seuls trois mécanismes peuvent théoriquement y conduire : une augmentation de la température, un abaissement de la pression ou une variation de composition. Sur ce dernier point, ce sont les fluides qui jouent un rôle déterminant. L'augmentation de température ne joue qu'un rôle négligeable en volcanologie. Avec la diminution de pression lors de la remontée à l'état solide du manteau, l'élément déterminant pour la fusion des roches est sa teneur en eau : l'addition de $1 \%$ seulement d'eau en poids abaisse la température de fusion de $100{ }^{\circ} \mathrm{C}$ environ [Jaupart C., et al., (2003)]. Ainsi, à température constante, un processus de fusion peut être simplement amorcé par apport d'eau ou par diminution de pression. Ce sont de très loin les deux mécanismes les plus importants.

\section{II.3. Un magma riche en eau}

Les gaz volcaniques émis par les volcans sont composés, par ordre d'importance, d'eau $\left(\mathrm{H}_{2} \mathrm{O}: 75\right.$ à $\left.95 \%\right)$, de gaz carbonique $\left(\mathrm{CO}_{2}: 5\right.$ à $\left.25 \%\right)$ et de dioxyde de soufre $\left(\mathrm{SO}_{2}: 3\right.$ à $25 \%$ ) [Bonin B. (2004), Sigurdsson H. (2000)] Viennent ensuite d'autres éléments volatils en beaucoup plus faible pourcentage : le monoxyde de carbone (CO), l'acide chlorhydrique $(\mathrm{HCl})$, l'hydrogène $\left(\mathrm{H}_{2}\right)$, l'hydrogène sulfuré $\left(\mathrm{H}_{2} \mathrm{~S}\right)$, l'acide fluorhydrique $(\mathrm{HF})$, le soufre $\left(\mathrm{S}_{2}\right)$, et l'hélium (He)... (cf. Figure 3). Ces gaz volcaniques vont être libérés dans des proportions équivalentes entre l'éruption et les phénomènes pré et postéruptifs (fumerolles, manifestations hydrothermales, etc.). Ils constituent un indicateur de grand intérêt dans le domaine de la prévision volcanique. Les gaz volcaniques sont en partie à l'origine de l'atmosphère et de sa composition actuelle.

Dans les processus magmatiques, parmi les éléments volatils, l'eau est le principal acteur, car le gaz carbonique et les autres gaz, moins solubles dans le magma, n'ont qu'une action minoritaire. Le gaz carbonique et le dioxyde de soufre ont cependant un impact significatif sur le climat lors d'éruptions majeures [Detay M., 2013]. Tous ces éléments volatils sont originaires des éléments constitutifs des roches du manteau (péridotites) à l'origine du magma primaire. Ils s'individualisent progressivement en fonction des conditions environnementales de pression et de température.

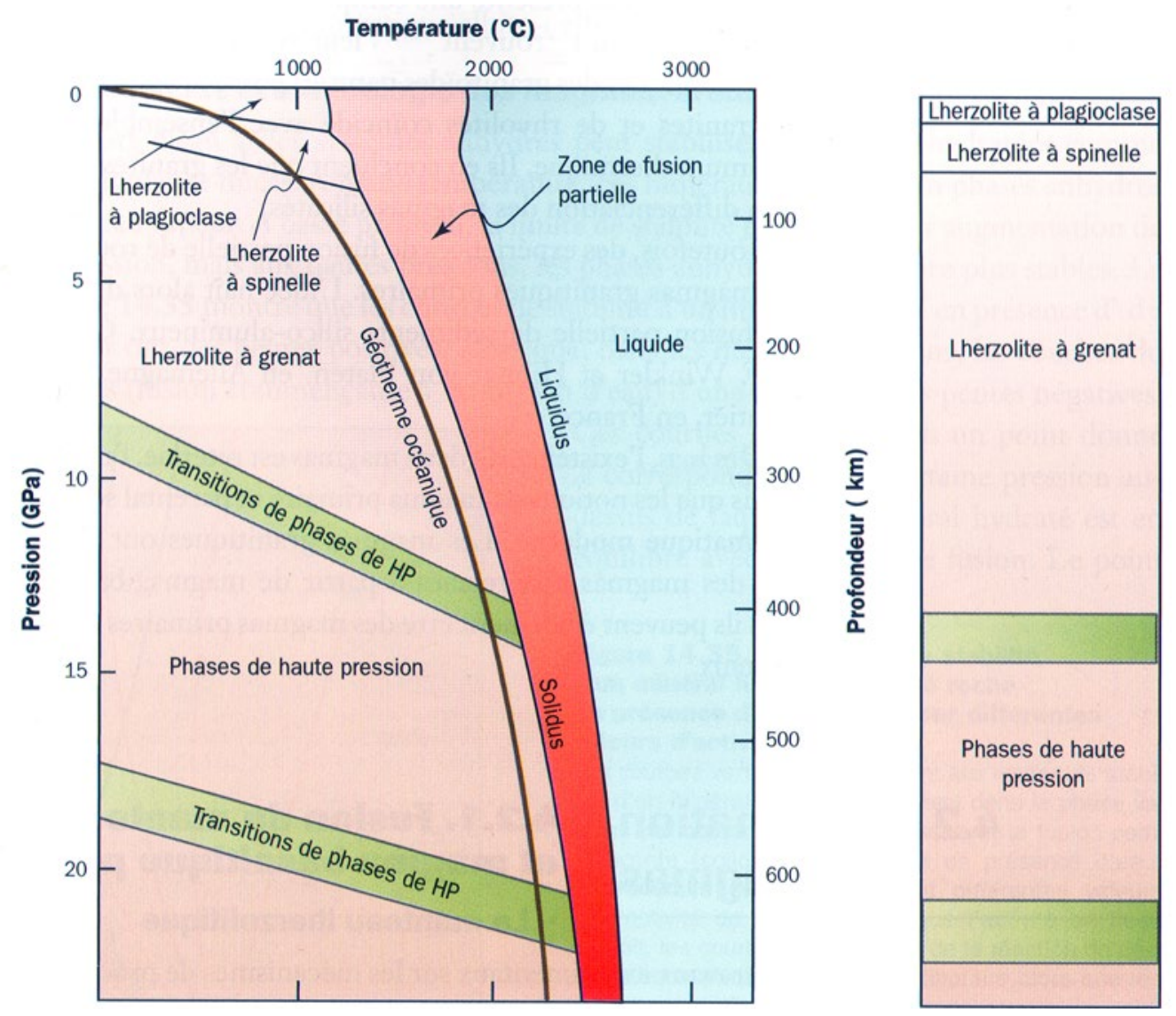

Figure 2 : Réactions subsolidus dans un manteau lherzolitique anhydre et section dans le manteau le long du géotherme considéré, d'après Robert C. et Bousquet R. (2013). 


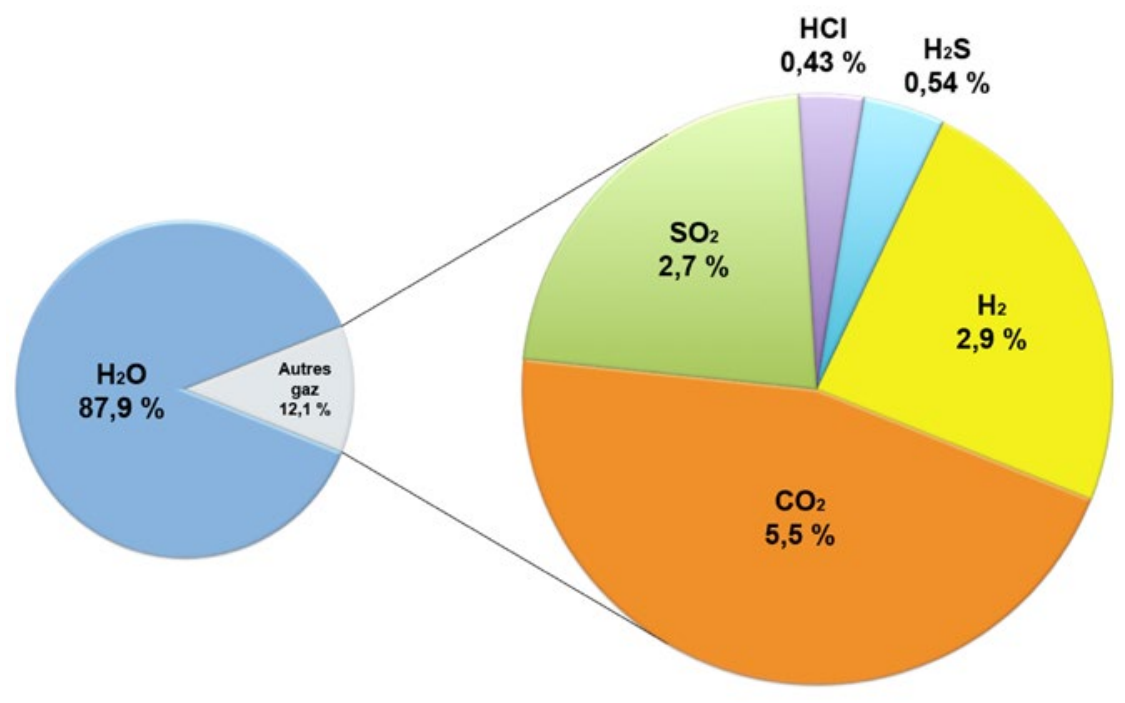

Figure 3 : Composition des gaz magmatiques, cas d'un basalte tholéitique de l'île de Surtsey (Islande). Les valeurs sont reportées en pourcentage de moles. L'eau est de loin le composant le plus abondant, suivie du gaz carbonique.

Les magmas terrestres peuvent renfermer jusqu'à $10 \%$ de leur poids en eau [Pichavant M., 2011]. Ces concentrations extrêmes se rencontrent dans les séries magmatiques des zones de subduction, mais des valeurs de 4 à $5 \%$ sont fréquentes. D'une manière générale, on trouve $1 \%$ d'eau dans des basaltes, 2 à $3 \%$ d'eau dans des andésites et jusqu'à $7 \%$ d'eau dans les rhyolites. L'eau a une influence considérable sur les propriétés physiques et chimiques des liquides silicatés (magmas). Après la fusion partielle, la présence d'eau va également avoir une action structurante sur l'ascension du magma, son dégazage, sa cristallisation et sa viscosité. Quelques pour-cent d'eau dans un magma font baisser sa viscosité de plus de six ordres de grandeur.

\section{HYDROVOLCANOLOGIE ÉRUPTIVE}

Lors du processus de fusion partielle dans le manteau terrestre, un liquide interstitiel s'individualise. On parle de fusion partielle, car seulement de 15 à $30 \%$ de la roche mère au maximum est susceptible de passer à l'état liquide. Plus chaude et moins dense que son milieu environnant, la phase liquide va remonter vers la surface par simple différence de masse volumique (principe d'Archimède). Le magma basaltique chaud et liquide a une masse volumique de 2700 à $2900 \mathrm{~kg} \cdot \mathrm{m}^{-3}$, celle d'une péridotite est d'environ $3300 \mathrm{~kg} \cdot \mathrm{m}^{-3}$. Cette ascension est généralement lente (quelques milliers d'années voire quelques centaines de milliers d'années), mais peut dans certains cas être très rapide (quelques mois dans le cas des éruptions expresses) [Ruprecht P., Plank T. 2013].

Pour que le magma se déplace " rapidement», il faut que sa viscosité le permette et qu'il puisse se frayer un passage à travers un réseau de fissures et de fractures plus ou moins interconnectées qui permettra son déplacement physique vers la surface. Ce sujet a été longtemps débattu par les scientifiques. Historiquement, le premier modèle proposé a été celui du " diapirisme », où une " poche » de magma, plus légère, sous la poussée d'Archimède, remonte en masse vers la surface comme la cire dans une lampe à lave (lava lamp). Le principal problème est de disposer de matériaux assez ductiles (encaissant) pour permettre la remontée du diapir. Plus récemment, dans les années 1980-1990, un nouveau modèle conceptuel a été proposé répondant au nom de dyking où les magmas, plutôt que de former de grosses boules, montent vers la surface grâce à un réseau de filons (dykes). Ces dykes servent de zone d'alimentation aux plutons superficiels proprement dits, qui se mettent en place, non pas en masse comme un diapir, mais plutôt comme un ballon qu'on gonfle peu à peu (ballooning). Cette théorie a été confortée par l'étude de plutons où l'on a pu mettre en évidence les dykes, véritables racines d'alimentation par le bas de la plomberie d'alimentation des réservoirs magmatiques. Une variante de ce modèle est maintenant basée sur la circulation et l'injection de magma non plus par des fractures cassantes, mais plutôt le long de zones de cisaillement (shear zones). Aujourd'hui, on penche pour des combinaisons de tous ces processus.

En remontant, le magma peut ralentir sa progression dans une ou plusieurs chambres magmatiques, la pression interne va être directement fonction de la teneur en eau d'un magma différentié. En profondeur, le magma primaire est sous-saturé en eau, car celle-ci est entièrement dissoute dans le liquide magmatique. En effet, compte tenu des gradients de pression et de température en profondeur, l'eau se comporte comme un fluide hypercritique, et il n'y a pas de discontinuité gaz-liquide. Par contre, en remontant, quand l'eau n'est plus en condition hypercritique, à moins de 218 bars, (220 bar $=22 \mathrm{MPa}$ soit $700 \mathrm{~m}$ de profondeur dans le sous-sol, $2200 \mathrm{~m}$ sous la mer), une phase gazeuse s'individualise (cf. Figure 4).

En se rapprochant de la surface, les éléments volatils prennent le relais de la poussée d'Archimède. En effet, les magmas contiennent des éléments volatils comme l'eau et le dioxyde de carbone. La solubilité de ces espèces est une fonction croissante de la pression. Dans la plupart des cas, ces éléments volatils sont dissous dans le magma aux fortes pressions qui règnent dans les zones où la fusion partielle est possible. La baisse de pression qui résulte de l'ascension conduit inévitablement à l'apparition d'une phase gazeuse [Jaupart C., Kaminski E. 2006]. Dans son parcours vers la surface, le magma primaire subit une décompression très 


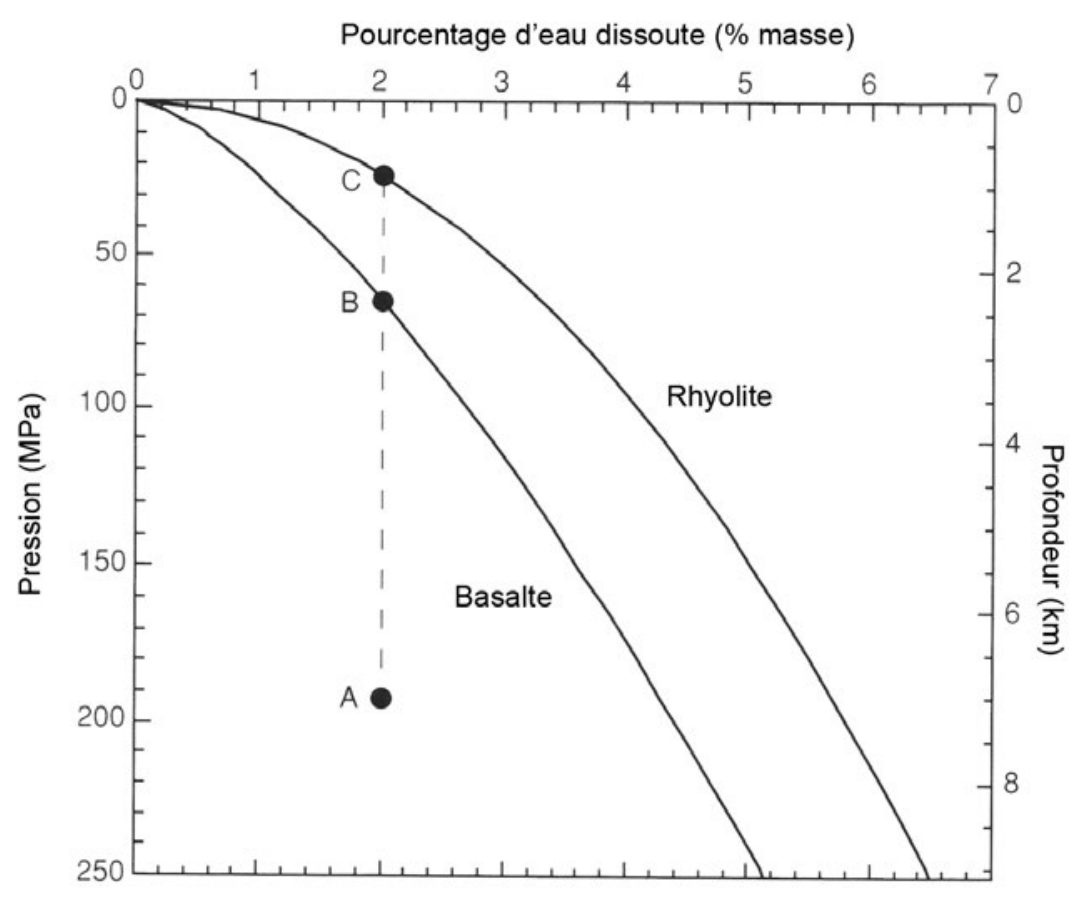

Figure 4 : Solubilité de l'eau (à $2 \%$ de la masse) dans les rhyolites et les basaltes en fonction de la profondeur (en l'absence d'autres éléments volatils dans le magma). Lors de la remontée du magma de A à B il devient saturé en eau si c'est un basalte ou au point $C$ s'il s'agit d'une rhyolite. Comme les deux magmas continuent leur parcours ascensionnel, ils deviennent sursaturés en eau respectivement au-delà de B et C. À ce point, l'eau exsolve et une phase gazeuse s'individualise. D'après Parfitt E. A. et Wilson L. (2008), modifié.

importante. L'origine des magmas primaires mantelliques dépend du contexte géodynamique, mais elle est généralement située à $100 \pm 50 \mathrm{~km}$. Une roche située à $100 \mathrm{~km}$ de profondeur subit une pression de $3 \mathrm{GPa}$, soit environ 30000 fois la pression atmosphérique. Lorsqu'un magma prend naissance à $100 \pm 50 \mathrm{~km}$ de profondeur et qu'il remonte et arrive en surface, il a subi une décompression d'un facteur 15000 à 45000 .

Lors de la remontée du magma, le dioxyde de carbone est la première espèce volatile à former des bulles dans les magmas, dès les profondeurs mantelliques. Il est suivi par l'eau, le soufre, puis les halogènes, dont les pressions initiales d'exsolution sont plus modérées, voire faibles, et fortement dépendantes de la nature et de l'évolution du liquide. Cette séquence d'exsolution va influencer le degré de cristallisation du magma, ainsi que son état redox, et donc la solubilité des espèces volatiles encore dissoutes. Le système est dynamique et se rééquilibre en permanence. La composition chimique de la phase gazeuse exsolvée évolue au cours de la décompression, entre un pôle profond dominé par le $\mathrm{CO}_{2}$ et un pôle superficiel dominé par l'eau.

\section{III.1. Nucléation}

La nucléation au sein d'un magma est un phénomène complexe. Elle correspond à une transition de phase par la formation d'une bulle au sein d'un liquide silicaté métastable. Le coût énergétique nécessaire à la création de l'interface magma-fluide supercritique peut différer le processus de nucléation. Il s'agit d'un processus microscopique au cours duquel des atomes d'une phase se réorganisent pour former un petit volume (germe, embryon, nucleus) d'une nouvelle phase, suffisamment gros pour être thermodynamiquement stable. Il existe une taille de bulle critique au-delà de laquelle la bulle va croître spontanément. En théorie, la nucléation devrait commencer dès que le magma se trouve saturé en une espèce volatile. Dans la nature, il faut un certain degré de sursaturation pour que le processus s'engage. En effet, la tension de surface du magma doit être vaincue pour que soit créé un nucleus. Un certain degré de cristallisation du magma aide à la nucléation dans la mesure où les cristaux constituent des irrégularités au sein du liquide magmatique, facilitant ainsi le déclenchement du processus (germes de nucléation, ils abaissent l'énergie interfaciale nécessaire entre magma et fluide supercritique). On distingue la nucléation homogène, lorsque la formation des nucléus résulte des fluctuations locales de la densité, d'origine thermique, de la nucléation hétérogène, qui est déclenchée par les impuretés du liquide ou la présence de parois. La nucléation dans un magma est généralement hétérogène car elle apparaît sur des sites de nucléation préférentiels (interfaces préexistantes, phases ayant préalablement nucléé, défauts de surface, impuretés, cristaux). Typiquement, l'énergie de sursaturation d'un volatil dans un magma est de quelques mégapascals dans le cas d'une nucléation hétérogène et de 10 à $100 \mathrm{MPa}$ pour une nucléation homogène.

\section{III.2. Diffusion, décompression, coalescence}

Dès que la nucléation est déclenchée, la diffusion prend le relais et devient le grand mécanisme responsable de l'augmentation de la taille des bulles dans le magma. Elle va assurer la migration des gaz dans la bulle néoformée à partir des éléments volatils présents dans le liquide magmatique.

La décompression joue également un rôle. La loi de Boyle-Mariotte énonce que :

$$
P_{1} V_{1}=P_{2} V_{2}
$$


où $P$ est la pression et $V$ le volume d'un gaz. Cela signifie que si la pression du gaz diminue, son volume augmente. Ainsi, quand du magma riche en bulles remonte vers la surface, sa pression diminue, donc le volume des bulles de gaz augmente. Si l'on prend l'exemple d'une bulle de gaz à $300 \mathrm{~m}$ de la surface qui va grossir par décompression, la pression $\left(P_{l}\right)$ qu'elle subit peut s'écrire :

$$
P_{1}=\rho g h
$$

où $(\rho)$ représente la masse volumique de magma, $(g)$ la pesanteur $\left(9,81 \mathrm{~m} \cdot \mathrm{s}^{-2}\right)$ et $(h)$ la hauteur. Si l'on retient $\rho=2800 \mathrm{~kg} \cdot \mathrm{m}^{-3}, P_{1}$ est donc de $8,25 \mathrm{MPa}$. Comme la pression à la surface $P_{2}$ est de 1 bar soit $0,1 \mathrm{MPa}$. Ainsi $V_{2}=(8,25 / 01) V_{1}$ soit 82,5 $V_{1}$. Mathématiquement, le volume d'une bulle est proportionnel au cube de son rayon. En effet, tant que la bulle ne remonte pas trop rapidement on peut l'assimiler à une sphère. On obtient donc $r_{2}=8,25^{1 / 3}=$ $4,35 r_{l}$. Ainsi, une bulle dans le magma, entre une profondeur d'environ $220 \mathrm{~m}$ et sa libération à la pression atmosphérique, va croître par décompression et diffusion d'un facteur 1000 environ. La décompression à elle seule va être responsable d'une augmentation du rayon par un facteur 4 environ, ce qui signifie, dans ce cas, que l'augmentation de taille est majoritairement contrôlée par la diffusion.

La coalescence ( $\mathrm{cf}$. Figure 5) est le troisième phénomène responsable de la croissance des bulles de gaz dans le magma. Ce phénomène est surtout structurant dans les éruptions stromboliennes et vulcaniennes. Les bulles de gaz sont toujours sous l'influence de la poussée d'Archimède et, du fait de leur faible densité, vont avoir tendance à remonter plus vite que le magma lui-même. Il se forme une mousse magmatique au sommet de la colonne ascendante. Toujours parce que la bulle est assimilable à une sphère, sa flottabilité $(F)$ peut s'écrire :

$$
F=\frac{4}{3} \pi r^{3}\left(\rho_{\text {magma }}-\rho_{g a z}\right) g
$$

où $(r)$ représente le rayon de la sphère, $(\rho)$ la masse volumique du magma et celle du gaz, et $(g)$ la gravité.

Tant que le rayon de la bulle n'est pas excessif, la force de traction est du même ordre de grandeur que la poussée d'Archimède, ainsi la vitesse de remontée $(v)$ de la bulle est :

$$
v=\frac{\left[\left(\frac{2}{9}\right)\left(\rho_{\text {magma }}-\rho_{g a z}\right) g r^{2}\right]}{\eta}
$$

où $(\eta)$ représente la viscosité du magma.

L'équation 4 met en évidence que la vitesse ascensionnelle d'une bulle dans le magma est proportionnelle au carré du rayon, plus une bulle est grosse plus elle va remonter rapidement.

La vitesse de remontée des bulles est inversement fonction de la viscosité du magma au sein duquel elles vont se déplacer. Pour fixer les idées, prenons l'exemple d'une bulle de $100 \mu \mathrm{m}$ de diamètre remontant dans un magma de masse volumique $\rho_{\mathrm{m}}=2500 \mathrm{~kg} \cdot \mathrm{m}^{-3}$. Dans un magma basaltique d'une viscosité de 30 Pa.s, la bulle va atteindre une vitesse de $1,8 \mu \mathrm{m} \cdot \mathrm{s}^{-1}$. Dans un magma rhyolitique d'une viscosité de $10^{5} \mathrm{~Pa}$.s, sa vitesse ascensionnelle va être 3000 fois moindre $\left(0,54 \mathrm{~nm} \cdot \mathrm{s}^{-1}\right)$. À l'opposé, une plus grosse bulle de $10 \mathrm{~mm}$ atteindra respectivement des vitesses de $18 \mathrm{~mm} \cdot \mathrm{s}^{-1}$ dans un magma basaltique et de $5,4 \mu \mathrm{m} \cdot \mathrm{s}^{-1}$ dans un magma rhyolitique. Ainsi, même si, quelle que soit la taille, les ordres de grandeur des vitesses ascensionnelles restent comparables $(\approx 1 / 3000)$, la distance parcourue en une seconde par la grosse bulle dans un magma basaltique est du même ordre que sa taille, alors que dans une rhyolite ce ratio sera de l'ordre d'un vingtième de pour-cent de sa taille $(\approx 1 / 1850)$ soit près de 2000 fois plus faible.

Dans le cas de magmas acides (riche en silice), la viscosité ne permet pas d'obtenir un différentiel de vitesse significatif entre les bulles et le magma (dans le temps de la décompression). De ce fait, lorsque deux bulles sont suffisamment (a)

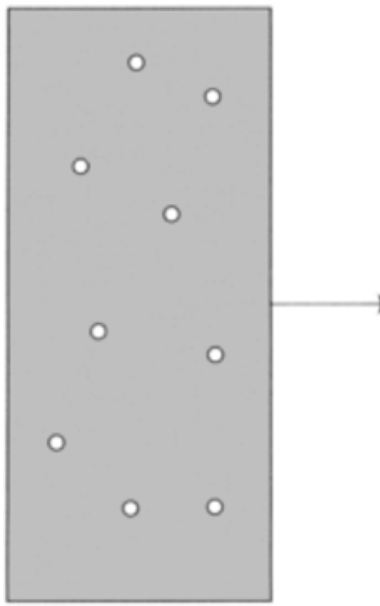

(b)

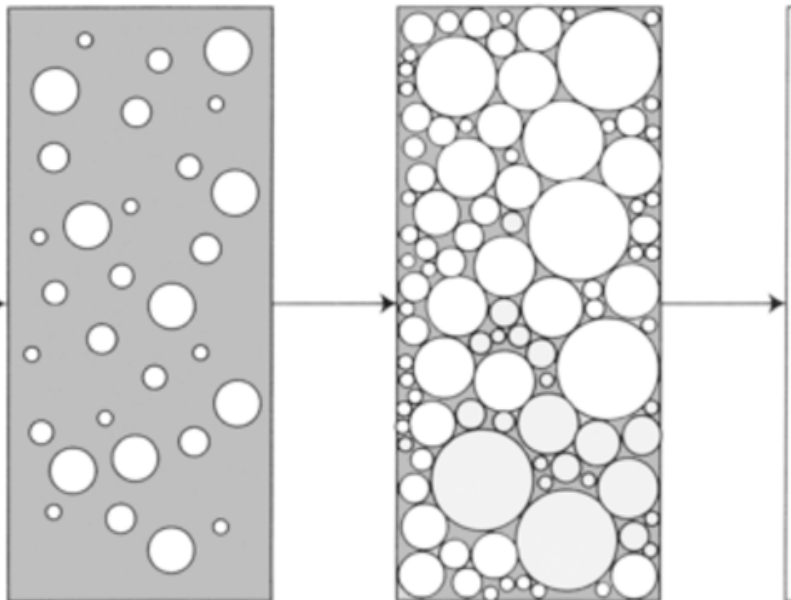

(d)

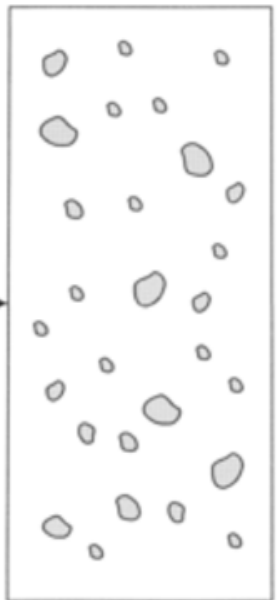

Figure 5 : Représentation schématique des quatre étapes du grossissement des bulles de gaz dans un magma, conduisant à la fragmentation. Les bulles commencent à se former en (a) : sursaturation. Elles se multiplient en (b) alors que la décompression s'accentue. Elles deviennent tellement nombreuses en (c) que l'espace interbulles commence à s'effondrer. En (d) le magma est passé de l'état de liquide contenant du gaz à un gaz contenant des gouttes de liquide. D'après Parfitt E. A. et Wilson L. (2008), modifié. 
rapprochées, et que leur taille est suffisamment grande, le film de liquide silicaté entre les bulles peut se rompre et amener les bulles à fusionner. Si l'on considère que la pression interne dans une bulle est égale à la pression externe ajoutée à la tension de surface à l'interface magma-fluide supercritique, il peut y avoir coalescence. Si deux bulles proches sont de taille équivalente, elles vont fusionner pour diminuer leur pression interne. Si les deux bulles proches sont de taille différente, la tension de surface de la petite bulle est supérieure à celle de la grosse bulle, la petite bulle aura donc tendance à diffuser vers la grosse bulle pour rétablir l'équilibre de pression. Ces processus jouent un rôle déterminant dans l'évolution de la perméabilité du magma au fur et à mesure de la vésiculation, puisque c'est par ce biais que les bulles peuvent former des chenaux ou des poches de gaz dans le magma, pouvant mener à la perte des volatils vers l'encaissant et à la fragmentation du magma. Ce phénomène d'emballement (runaway) peut amener à ce qu'une seule bulle occupe toute la taille du conduit éruptif (poches de gaz ou slugs). C'est ce mécanisme qui est responsable des éruptions stromboliennes, mais aussi du gas-piston effect qui fait parfois osciller les lacs de laves au Kilauea à Hawaï, au Nyiragongo en République Démocratique du Congo (RDC).

\section{III.3. Exsolution-fragmentation et mousse magmatique}

Le phénomène de fragmentation est très important, car c'est lui qui détermine le régime de l'écoulement, aussi bien dans le conduit éruptif que dans l'atmosphère. Dans le conduit éruptif, c'est lui qui marque le passage entre l'écoulement laminaire du magma chargé de bulles et l'écoulement turbulent de gaz chargé de fragments. Après la sortie dans l'atmosphère, c'est toujours lui qui détermine si l'éruption se développe en colonne plinienne ou en coulée pyroclastique (courant de densité pyroclastique - $C D P$ ). En volcanologie, la fragmentation désigne le processus (dégazage) qui transforme le magma en une mousse magmatique. Le liquide magmatique répond alors à un comportement biphasique (liquide-gaz) voire polyphasique, s'il y a également des cristaux.

Le concept de mousse magmatique (foam) [Vergniolle S., Jaupart C. 1990], obtenu après des expériences analogiques et la formulation mathématique du phénomène, permet notamment d'expliquer l'évolution d'une dynamique effusive de type fontaines de lave à celle d'une éruption strombolienne, l'effondrement de la couche de mousse magmatique pouvant produire une très grosse bulle (slug) dans le conduit magmatique. Pour cela il faut une assez grosse chambre magmatique dont la surface du toit est d'au moins $8 \mathrm{~km}^{2}$, et des bulles de 0,1 à $0,6 \mathrm{~mm}$; ce qui est le cas à Hawaï. Schématiquement, la mousse magmatique se forme par diffusion, décompression, coalescence et s'accumule au sommet de la chambre magmatique par différence de densité (Archimède, flottabilité). La chambre magmatique va alors se vidanger par l'intermédiaire du conduit magmatique. La tuyauterie magmatique (conduit) est également un réacteur au sein duquel la mousse magmatique va évoluer. L'épaisseur de l'horizon de mousse magmatique est proportionnelle à :

$$
\left(\frac{\eta_{\text {magma }} Q}{\varepsilon^{2} \rho_{\text {magma }} g}\right)^{1 / 4}
$$

où $\left(\eta_{\text {magma }}\right)$ et $\left(\rho_{\text {magma }}\right)$ représentent la viscosité et la densité du magma, $(\varepsilon)$ est la fraction de gaz contenue dans la mousse, $(g)$ la gravité et $(Q)$ le débit de gaz.
Les bulles vont se déformer dans le conduit magmatique sous l'action de la poussée d'Archimède. Il existe alors une épaisseur limite $\left(h_{c}\right)$ de mousse magmatique telle que :

$$
h_{c}=\frac{2 \sigma}{\varepsilon \rho_{\text {magma }} g r}
$$

où $(\sigma)$ est le coefficient de tension de surface et $(r)$ le rayon initial de la bulle. Si cette épaisseur critique est atteinte, la mousse s'effondre sous la forme d'une grosse bulle de gaz (slug) qui va remonter dans le conduit magmatique et exploser à la surface (éruption strombolienne). À l'opposé, si l'épaisseur de la mousse magmatique reste supérieure à sa valeur son épaisseur limite, on assiste à une éruption effusive accompagnée de fontaines de lave (éruption effusive).

L'effondrement de la mousse est ainsi sous la dépendance de trois variables : la viscosité, la tension de surface et la taille de la bulle. Le passage d'un régime à l'autre apparaît généralement quand le flux de masse est inférieur à $10^{3} \mathrm{~kg} \cdot \mathrm{s}^{-1}$. La mousse magmatique présente une perméabilité qui va permettre à une fraction de gaz de s'échapper. Les études théoriques et les travaux en laboratoire montrent que les magmas deviennent perméables autour d'une porosité de $30 \%$, quand les bulles commencent à croître par coalescence. La perméabilité augmente rapidement quand le magma remonte vers la surface, et on observe des perméabilités de l'ordre d'environ $10^{-12} \cdot \mathrm{m}^{2}$ pour une mousse magmatique comprenant de 60 à $70 \%$ de vésicules. Les magmas basaltiques perdent leur gaz plus facilement car ils ont une viscosité faible ce qui conditionne des éruptions effusives, moins violentes que les explosives. Cependant les bulles de gaz peuvent croître par décompression et diffusion pendant l'ascension du magma basaltique, mais elles sont trop petites pour se séparer et former une mousse magmatique. La coalescence peut alors permettre d'obtenir des bulles de tailles suffisantes pour que les gaz s'échappent pendant l'ascension du magma. C'est ainsi que l'on observe des fontaines de laves et des styles stromboliens en présence de laves basaltiques. Dans de nombreux cas, les deux styles éruptifs s'alternent ou coexistent. Ces variations brutales de style sont liées au dégazage du magma et à la stabilité ou l'instabilité de la mousse magmatique.

\section{III.4. Le déclenchement de l'éruption}

Le volume occupé par les gaz volcaniques est directement sous le contrôle de la pression (équation 1) : pour un magma contenant de 3 à $6 \%$ d'eau, on calcule qu'à $3000 \mathrm{~m}$ de profondeur $\left(10^{8} \mathrm{~Pa}\right)$, dans la chambre magmatique, des éléments volatils occupant $1 \%$ du volume vont subir une décompression progressive jusqu'à en occuper $91 \%$ à la pression atmosphérique.

La Figure 6 retrace la vitesse de remontée du magma dans un dyke. Le magma remonte de façon assez lente et constante jusqu'au point $\mathrm{A}$ où le phénomène de nucléation démarre. L'expansion des bulles de gaz fait accélérer le magma jusqu'au point $B$ où la fragmentation prend le relais. Au-delà de $\mathrm{B}$, les forces de friction contre les parois du dyke (pertes de charge) deviennent négligeables, et le magma prend encore de la vitesse jusqu'au point $\mathrm{C}$ où il provoque une éruption pyroclastique en arrivant à la surface.

Un faible niveau de sursaturation $(<1 \mathrm{MPa})$ suffit pour initier la nucléation. Les bulles croissent alors que la pression décroît. Leur croissance est cependant limitée à environ $64 \%$ du volume de magma, seuil à partir duquel on 


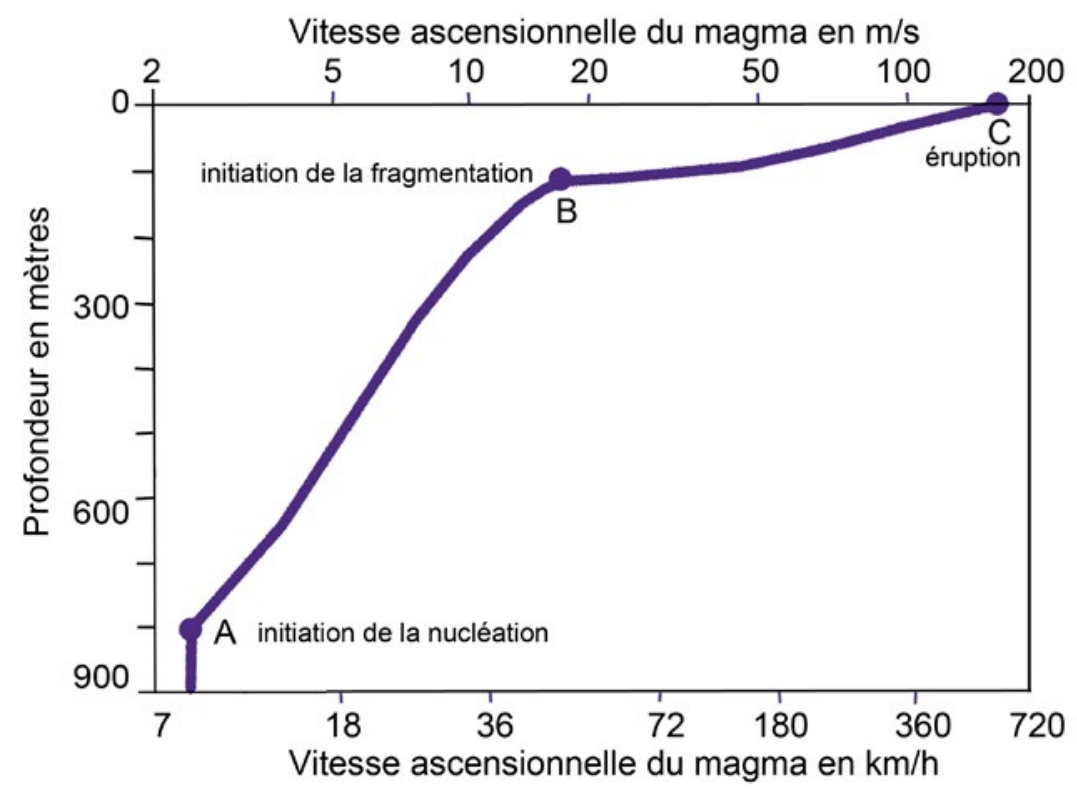

Figure 6 : Vitesse ascensionnelle du magma dans un dyke, influence de l'exsolution des éléments volatils.

passe d'un liquide contenant des bulles (magma) à un gaz contenant du liquide $(C D P)$. La vitesse ascensionnelle du magma proche du cratère peut atteindre des centaines de mètres par seconde. Par exemple, lors de l'éruption du mont St Helens, la vitesse ascensionnelle était supérieure à $344 \mathrm{~m} \cdot \mathrm{s}^{-1}$. À l'inverse, dans des magmas basaltiques très fluides (à Hawaï, en Islande ou à La Réunion), qui contiennent très peu d'eau $(0,45 \%$ de sa masse pondérale), la nucléation ne commence qu'à $300 \mathrm{~m}$ de la surface, ce qui génère quand même des fontaines de lave de plus de $500 \mathrm{~m}$ de hauteur. La teneur en volatils des magmas impose ainsi toujours un style éruptif explosif, même si ce dernier n'est que de courte durée. Le style effusif pur n'existe donc pas pour des volcans émergés. Il peut au mieux caractériser une phase de l'éruption après que les gaz ont été largement libérés [Detay M., Thomas P. 2014].

Dans les cas de forte surpression interne, le toit de l'édifice volcanique peut être littéralement pulvérisé comme ce fut le cas pour le Pinatubo "décapité 》 de ses $145 \mathrm{~m}$ sommitaux lors de l'éruption de 1991 ; le mont St Helens, qui a perdu $430 \mathrm{~m}$ lors de l'éruption du 18 mai 1980 ; le Vésuve, qui a perdu $470 \mathrm{~m}$ lors de l'éruption de 1631, ou encore le Tambora, dont les $1500 \mathrm{~m}$ sommitaux ont été pulvérisés lors de l'éruption de 1815.

\section{III.5. De la versatilité de l'eau}

$\mathrm{Au}$ cours du processus de cristallisation fractionnée, des minéraux hydroxylés (amphiboles, micas) se forment en fixant l'eau sous forme $\mathrm{HO}^{-}$. Les amphiboles, par exemple, sont stables dans une gamme de pression de 2 à $3.10^{9} \mathrm{~Pa}$ (20 kbar à $25 \mathrm{kbar}$ ). Elles contiennent environ $2 \%$ d'eau. Lors de la cristallisation des amphiboles, le magma résiduel s'appauvrit alors en eau. Lorsque les conditions thermodynamiques évoluent, les amphiboles sont susceptibles de devenir instables soit pendant la remontée (baisse de la pression) soit à l'occasion d'une augmentation de la pression ou de température liée à une réalimentation magmatique. Les amphiboles libèrent alors leur eau. Cet « effet amphibole » [Bardintzeff J.-M., Bonin B. 1986, Bardintzeff J.-M. 2011] joue ainsi un rôle important dans l'apport d'eau aux magmas. Des travaux expérimentaux montrent que cette libération se produit lors de la remontée du magma entre 8000 à $5000 \mathrm{~m}$ de profondeur environ. Les micas jouent un rôle similaire. Ils intègrent dans leur structure $4 \%$ d'eau. Ces minéraux peuvent donc jouer un rôle critique, notamment s'ils se résorbent lors de l'ascension du magma. Ils libèrent alors une quantité d'eau importante qui, en passant en phase vapeur, est susceptible d'engendrer des surpressions considérables pouvant déclencher une éruption.

Lors de la remontée du magma, l'exsolution est responsable de changements structurants des propriétés physico-chimiques du magma : variation de densité, de viscosité, changement dans l'équilibre de diverses phases, initiation de la cristallisation, ... La compressibilité d'un magma riche en gaz (mousse magmatique) contribue également à compliquer significativement le mode d'écoulement du magma dans la plomberie magmatique (écoulement biphasique, polyphasique, Newtonien, Binghamien, viscoélastique...), et l'exsolution des gaz [Fagent S.A. et al. 2013]. L'ensemble de ces phénomènes concourt à la grande diversité des phénomènes éruptifs qui sont susceptibles de se succéder lors d'un seul et même événement. Les éruptions volcaniques sont donc gouvernées par des lois complexes, non linéaires, voire chaotiques, responsables de phénomènes éminemment instables. Les éruptions volcaniques sont donc intrinsèquement imprévisibles.

\section{III.6. L'eau et les styles éruptifs}

La teneur en éléments volatils des magmas, et en eau en particulier, permet de rendre compte des divers styles éruptifs que l'on observe sur Terre. Des fontaines de lave (style hawaïen) aux éruptions explosives vulcaniennes ou pliniennes. Dans tous les cas, ce sont les éléments volatils libérés en surface par exsolution qui conditionnent des styles éruptifs plus ou moins violents. Lorsque le magma est très fluide et qu'il se dégaze facilement on obtient des fontaines de lave. Quand le magma est plus visqueux et que la chambre volcanique est de taille suffisante, un volume de 
gaz (slug) va pouvoir s'individualiser et entraîner un style strombolien. Quand les éléments volatils se trouvent dans un magma plus visqueux et acide ils vont imposer des styles explosifs plus ou moins violents : de vulcanien à plinien, par exsolution-fragmentation.

L'eau est donc un élément d'importance en volcanologie. L'hydrovolcanologie, discipline à l'interface des sciences hydrotechniques et de la volcanologie, a pour ambition de mieux comprendre ces phénomènes.

\section{HYDROVOLCANOLOGIE SUPERFICIELLE}

Les gaz volcaniques sont libérés à $50 \%$ lors de l'éruption et à $50 \%$ lors des phénomènes pré et postéruptifs. Ainsi l'étude des gaz, dont l'eau représente la très grande majorité, est d'une importance cruciale en matière de prévision.

L'eau et le feu sont intimement liés depuis la formation du système solaire, il y a 4,5 milliards d'années [Detay M. (2004)]. La molécule d'eau participe, sous diverses formes, au magmatisme (plutonisme et volcanisme). Elle est notamment fondatrice de la phase hydrothermale responsable des évents hydrothermaux sous-marins comme des manifestations paravolcaniques superficielles (fumerolles, geysers, solfatares, lacs de cratère...). L'eau a principalement une origine superficielle. Les eaux profondes juvéniles, restent minoritaires $(<10 \%)$.

\section{IV.1. Solfatares et lacs acides}

Le catalogue des volcans actifs dans le monde [Siebert L. et al., 2010] estime le nombre de lacs acides à 769 , toutes positions géomorphologiques confondues. Bien que leur répartition soit mondiale, on les rencontre majoritairement associés au volcanisme d'arc. Les paramètres physico-chimiques de ces lacs sont extrêmement variables, car ils sont le reflet de l'activité hydrothermale et magmatique sous-jacente (cf. Figure 7). Pour rester relativement stables dans le temps, ces lacs ont besoin d'apports en eau constants, des berges imperméables, un flux géothermique et un apport significatif en éléments volatils. Cette dynamique est fréquente sur les volcans actifs où le dégazage est important. Le lac de cratère se comporte comme un réacteur où différentes réactions physico-chimiques (et biologiques) complexes vont opérer en parallèle. On rencontre des lacs dans les cratères, mais aussi dans des caldeiras d'effondrement et dans des cratères latéraux, produits du phréatomagmatisme qui peuvent constituer des maars.

Dans les régions tectoniquement actives comme le rift est-africain, les circulations hydrothermales peuvent apporter des sels dissous qui modifient sensiblement la chimie des lacs. C'est notamment le cas du lac Kivu et du lac Albert, dont la teneur en $\mathrm{Mg}^{2+}, \mathrm{K}^{+}$et $\mathrm{Cl}^{-}$se trouvent être 20 fois plus élevées que les concentrations du lac Victoria voisin. Ces concentrations « anormales » sont la signature de la présence du rift est-africain et d'apports hydrothermaux profonds.

\section{IV.2. Éruptions limniques}

Un autre effet du volcanisme est de produire du gaz carbonique en quantité importante. Ce gaz peut se mélanger à l'eau jusqu'à atteindre des concentrations extrêmes avant d'être relargué brutalement.

Les lacs acides des volcans actifs sont généralement trop acides pour conserver le $\mathrm{CO}_{2}$ sous forme d'ions bicarbonates $\left(\mathrm{HCO}_{3}^{-}\right)$à l'exception des lacs Nyos et Monoun (Cameroun), où les émissions de gaz acides ont cessé $\left(\mathrm{SO}_{2}, \mathrm{H}_{2} \mathrm{~S}, \mathrm{HCl}\right)$ ont cessé, faisant de ces lacs des réservoirs à sulfates et chlorures, mais surtout à $\mathrm{CO}_{2}$ dissous. Ce dernier est susceptible de passer à l'état gazeux, comme en attestent les éruptions limniques du Nyos (environ $300 \mathrm{Mm}^{3}$ de $\mathrm{CO}_{2}-1746$ morts en 1986) et du Monoun (environ $10 \mathrm{Mm}^{3}$ de $\mathrm{CO}_{2}-37$ morts en 1982). Suite à ces événements catastrophiques, ces deux lacs sont maintenant équipés de dispositifs artificiels de dégazage.

Le lac Kivu, situé en RDC, à 1463 m d'altitude, est le plus petit des Grands Lacs africains, soulignant le tracé du

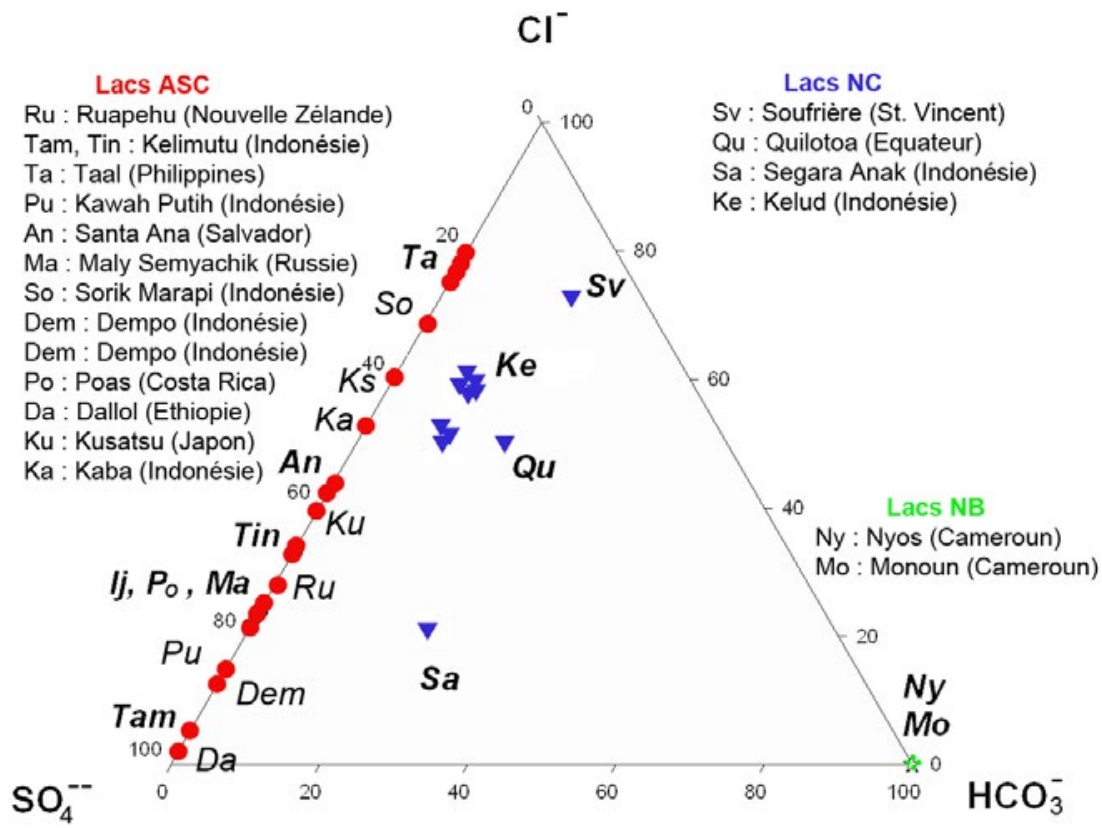

Figure 7 : Classification des lacs volcaniques en fonction de la concentration en anions. Type acide-sulfate-chlorure (ASC), neutre-chlorure (NC) et neutre-bicarbonate (NB) d'après Bernard A. (2004) complété et modifié, Detay M. (2012). 
rift est-africain. Les relargages observés dans le lac Kivu lors de l'épisode éruptif du Nyiragongo, en 2002, ont alerté les scientifiques sur le risque d'éruption limnique. En effet, ce lac a la caractéristique de renfermer de grandes quantités de gaz dissous $\left(\mathrm{CO}_{2}\right.$ et $\left.\mathrm{CH}_{4}\right)$ stratifiés dans ses profondeurs. Après l'éruption du Nyiragongo, des investigations ont permis de quantifier la taille des réservoirs à $300 \mathrm{~km}^{3}$ (soit $300000 \mathrm{Mm}^{3}$ ) de gaz carbonique et $55 \mathrm{~km}^{3}$ de méthane (quantifiés à $0{ }^{\circ} \mathrm{C}$ et une atmosphère) [Detay M. 2011]. Les catastrophes humaines qui ne manqueront malheureusement pas de se produire soulignent le risque que représente le lac Kivu, étant donné qu'entre un $\mathrm{km}^{3}$ (Kivu) et un $\mathrm{Mm}^{3}$ (Nyos, Manoun) il y a un facteur de $10^{3}$ c'est-à-dire que l'on parle du relarguage potentiel de 300 milliards de mètres cubes de $\mathrm{CO}_{2}$ pour le lac Kivu. Chaque litre d'eau du lac Kivu renferme 2 litres de gaz $(1 / 6$ de méthane et $5 / 6$ de gaz carbonique) soit 1000 fois plus de gaz qu'à Nyos ! Est-il utile de rappeler que le gaz carbonique, plus lourd que l'air, est incolore et inodore ? Sa toxicité commence à $3 \%$; un taux de $15 \%$ entraîne une perte brutale de connaissance ; à $25 \%$ il provoque un arrêt respiratoire qui entraîne le décès. De même, le méthane est incolore et inodore. Il peut, comme le $\mathrm{CO}_{2}$, provoquer des asphyxies en prenant la place de l'oxygène dans l'air. Il est par ailleurs extrêmement inflammable.

\section{HYDROVOLCANISME}

Le terme d'hydrovolcanisme s'emploie lors d'interactions entre magma et eau souterraine (aquifère) ou superficielle (lac, rivière, glacier, mer). Il convient de distinguer le phréatisme, lorsque l'eau surchauffée par le magma est la seule responsable de l'éruption, et le phréatomagmatisme lorsqu'il y a rencontre et mélange physique entre le magma et l'eau (cf. Figure 8). Dans une éruption phréatique on ne retrouve pas de magma frais, c'est uniquement l'eau souterraine qui a été surchauffée par transfert d'énergie, en profondeur, par des dykes remplis de magma. L'eau en passant à l'état gazeux finit par faire exploser l'encaissant sus-jacent quand sa pression interne devient supérieure à la pression externe (encaissant). La partie sus-jacente de l'édifice volcanique est généralement pulvérisée en cendres, lapilli et blocs. Ces éruptions explosives sont meurtrières par la balistique des blocs projetés et par les CPD qu'elles peuvent générer, comme cela vient d'être le cas au Japon (éruption de l'Ontake-san du 27 septembre 2014, provoquant la mort d'environ 70 personnes).

Généralement les éruptions phréatiques sont annonciatrices d'éruptions phréatomagmatiques, comme pour l'éruption du mont St Helens, susmentionnée, ou encore celle du Krakatau en 1883.

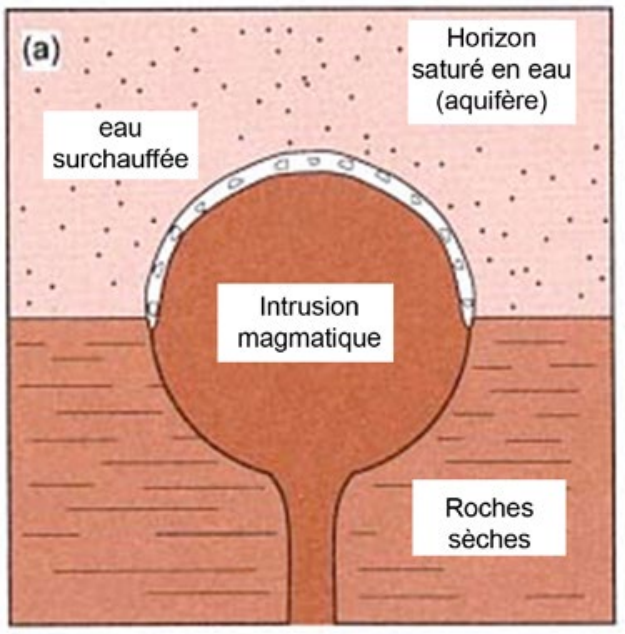

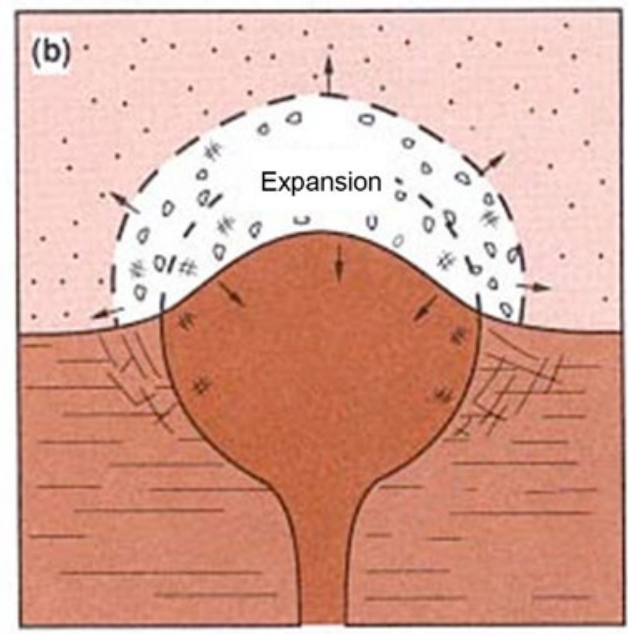

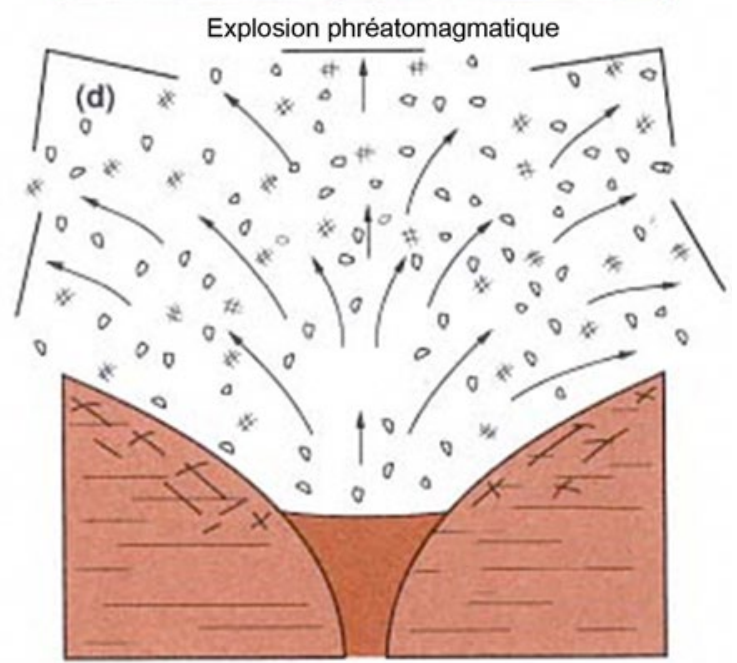

Figure 8 : Schéma conceptuel d'interaction magma-eau: (a) contact initial magma-aquifère, (b) création d'une zone hypercritique au contact magma-eau, (c) mélange magma-encaissant (aquifère), (d) expansion du mélange magma-eau-roche à haute pression et explosion de l'encaissant (éruption phréatomagmatique). Adapté d'après Sheridan M.F. et Wohletz K.H. (1981), modifié. 
L'éruption de l'Eyjafjöll qui a bloqué l'espace aérien européen en avril 2010 était la conséquence du contacte magma-glacier conférant à la seconde phase de l'éruption un caractère hydrovolcanique.

Il existe également un hydrovolcanisme sous-marin. Si le plancher est peu profond $(<1000 \mathrm{~m})$ on assiste à la naissance d'une île (cas de Surtsey en Islande, en novembre 1963, ou de Niijima, au large du Japon, en novembre 2013).

\section{HYDROVOLCANOLOGIE PRÉVISIONNELLE}

La compréhension des mécanismes magmatiques et le développement d'outils de mesure dédiés à l'étude des volcans ont permis des avancées significatives dans la prévision des éruptions volcaniques. Pour les volcans les plus dangereux (Vésuve, montagne Pelée, Hawaï, Nyiragongo, notamment), des observatoires volcanologiques ont vu le jour dans de nombreux pays. Une instrumentation a été mise en place et des scientifiques analysent les signaux en temps réel. La France a établi trois observatoires sur les édifices présentant des risques notamment à La Réunion (piton de la Fournaise), à la Guadeloupe (Soufrière de la Guadeloupe), et à la Martinique (montagne Pelée). De même pour l'aviation des centres de surveillance aéronautiques volcanologiques (Volcanic Ash Advisory Centers - VAAC) ont été mis en place (dont un en France à Toulouse).

Dans un premier temps, les observations de terrain donnent des indications quant à l'activité magmatique : intensité de l'activité solfatarienne et fumerollienne ; déformations du sol (éboulements, glissements, dilatation ou affaissement de l'édifice, variation de pente...) ; montée du niveau de l'eau dans les lacs ou changement de couleur ou encore apparition de bulles ; attitude des animaux ; activité sismique et microsismique ; activité sonore du volcan... Ces indications demandent à être complétées par des données quantifiables issues d'une instrumentation : analyse en continu des gaz, mesure de la température des solfatares..

\section{CONCLUSIONS}

L'étude des fluides géologiques permet de mieux comprendre le rôle central joué par les éléments volatils, et l'eau en particulier, en volcanologie. Elle révèle qu'ils représentent le principal moteur des phases superficielles de l'éruption. Plus que tout autre constituant, la molécule d'eau modifie en profondeur les propriétés physiques et chimiques des magmas. L'eau joue un rôle majeur en abaissant le solidus et en permettant la fusion partielle des péridotites du manteau à l'origine des magmas primaires dans les zones de subduction, là où il $\mathrm{y}$ a le plus de volcans émergés. Pendant sa phase ascensionnelle l'exsolution de l'eau influe sur la dynamique des magmas, son dégazage, sa cristallisation, sa viscosité, et conditionne tous les styles éruptifs aériens ou sous-marins peu profonds. Les styles éruptifs, contrairement à une idée ancienne, ne rendent compte, au mieux, que d'un moment particulier d'une éruption, de nombreux styles pouvant s'enchaîner au cours d'une seule et même phase éruptive.

Ce court article illustre la nécessité d'un travail d'équipe dans les sciences de la Terre et les sciences hydrotechniques. Le volcanologue doit jongler avec le travail de terrain, la mécanique des fluides géologiques et les phénomènes de fragmentation qui constituent l'hydrovolcanologie. Il illustre aussi le nécessaire aller et retour entre théorie et observations de terrain. La valeur d'une théorie ne réside pas uniquement dans son aspect quantitatif, mais aussi dans sa capacité à orienter les stratégies de mesure sur le terrain et de prévention pour les quelques 500 à 600 millions de personnes qui vivent aujourd'hui sous la menace d'un volcan.

Notre exposé ne peut malheureusement pas être exhaustif et nous ne pouvons pas couvrir tous les aspects des interactions eau-volcan dans un court article. D'autres aspects comme les écoulements des courants de densité pyroclastiques, les lahars, ou encore le volcanisme planétaire auraient pu être développés et venir le compléter.

\section{NOMENCLATURE}

$\begin{array}{lll}\text { Giga } & \mathrm{G} & 10^{9} \\ \text { Méga } & \mathrm{M} & 10^{6} \\ \text { Kilo } & \mathrm{k} & 10^{3}\end{array}$

Attention à l'utilisation de l'abréviation $\mathrm{km}^{3}$. Le kilomètre au cube n'est pas égal à un "kilo » mètre-cube même si l'abréviation est la même : le $\mathrm{km}^{3}$ n'est pas égal à $10^{3} \mathrm{~m}^{3}$, mais $10^{9} \mathrm{~m}^{3}$ soit un $\mathrm{Gm}^{3}$.

\section{REFERENCES}

\section{BardinTZeFf J.-M. (2011) - Volcanologie, Dunod éd. 313 p}

BardintZeFf J.-M., Bonin B. (1986) - L'effet amphibole : des dômes aux nuées ardentes. Comptes rendus de l'Académie des sciences. 303(2) : 159-162

Bonin B. (2004) - Magmatisme et roches magmatiques. Dunod éd. $301 \mathrm{p}$

Bolfan-Casanova N. (2005) - Water in the Earth's mantle. Mineralogical Magazine. 69(3) : 229-257

Detay M., Thomas P. (2014) - Le dôme de lave du Paluweh (ou Rerombola, Indonésie) : mise en place, effondrements, nuées ardentes et autres courants de densité pyroclastiques. Article en ligne sur Planet-Terre : http://planet-terre.ens-lyon.fr/article/ volcan-Paluweh-Indonesie.xml

Detay M. \& A-M. (2013) - Volcans. Du feu et de l'eau. Belin éd. $208 \mathrm{p}$

Detay M. (2013) - Impact du volcanisme sur le climat passé et présent de la Terre. LAVE. $162: 19-31$

DETAY M. (2012) - Hydrovolcanologie appliquée à la phase hydrothermale. LAVE. 159 : 14-27

DetAY M. (2011) - Le Nyiragongo : volcan de tous les dangers et maîtrise des risques. LAVE. $153: 16-29$

Detay M. (2010) - Éruption de l'Eyjafjöll, un volcan qui a du panache. Pour la Science. 392 : 70-76

Detay M. (2004) - Origine contingente de l'eau sur Terre. Éléments de synthèse déduits des données géologiques, géochimiques et des modèles astronomiques et planétologiques. $\mathrm{La}$ Houille Blanche. 1 : 88-100

Dewaele A., Sanloup C. (2005) - L'intérieur de la Terre et des planètes. Belin éd. $181 \mathrm{p}$

Fagents S.A., GregG T.K.P., Lopes R.M.C. (2013) - Modeling volcanic processes - the physics and mathematics of volcanism. Cambridge University Press Ed. $421 \mathrm{p}$

Jaupart C., Guiraud M., Komorowski J.-C., Sautter V., Boudon G., Parodi G.-C., Leyrit H., Lenat J.-F., Bardintzeff J.-M. (2003) - Le volcanisme, cause de mort et source de vie. Vuibert - Muséum d'Histoire Naturelle Ed. 328 p 
JAUPART C., KAMINSKi E. (2006) - Les éruptions volcaniques « explosives » : des grandes aux petites échelles. Bulletin de la Société Française de Physique. 152 : 5-10

Jolivet L. (2011) — L'eau et la dynamique lithosphérique. Géosciences. $13: 16-23$

PARfitT E. A., WiLson L. (2008) - Fundamentals of physical volcanology. Blackwell Publishing. 230 p.

Pichavant M. (2011) — De l'eau dans les magmas. Géosciences. $13: 24-29$

Robert C., Bousquet R. (2013) - La dynamique du système Terre. Géosciences - Belin Ed. 1159 p

Ruprecht P., Plank T. (2013) - Feeding andesitic eruptions with a high-speed connection from the mantle. Nature. 500 : 68-72
SHERIDAN M.F., WoHLETz K.H. (1981) - Hydrovolcanic explosions: the systematics of water-pyroclast equilibration. Science. 212 : 1387-1389

Schmandt B., Jacobsen S.D., Becker T.W., Liu Z., Dueker K. G. (2014) - Dehydration melting at the top of the lower mantle. Science. 344 (6189) : 1265-1268

Siebert L., Simkin T., Kimberly P. (2010) - Volcanoes of the world. Smithsonian - University of California Press. $551 \mathrm{p}$

Sigurdsson H. (2000) - Encyclopedia of volcanoes. Academic Press. $1417 \mathrm{p}$

Vergniolle S., Jaupart C. (1990) - Dynamics of degassing at Kilauea volcano, Hawaii. Journal of Geophysical Research. 95 : 2793-2809 